\title{
Improving Efficiency of a Genetic Algorithm Applied to Multi-robot Tactic Operation
}

\author{
Gustavo Pessin, Fernando S. Osório, Denis F. Wolf, and Christiane R.S. Brasil \\ University of São Paulo (USP) \\ Institute of Mathematics and Computer Science (ICMC) \\ Av. Trabalhador Sãocarlense, 400 - C.P. 668 - 13.560-970 - São Carlos, SP \\ \{pessin,fosorio, denis, christiane\}@icmc.usp.br
}

\begin{abstract}
There are two important issues in the Genetic Algorithm searching and optimization process: population diversity and selective pressure. These two issues are strongly related and have direct impact on the search efficiency. Two factors that directly influence these issues are often ignored: overlapping populations and fitness scaling. In this paper we address the use of overlapping populations and fitness scaling in a Genetic Algorithm (GA) applied to multi-robot squad formation and coordination. The robotic task is performed over a natural disaster scenario (a forest fire simulation). The robot squad mission is surrounding the fire and avoiding fire's propagation based on the strategy proposed by the GA. Simulations have been carried out with several GA parameters (several types of scaling and different degrees of overlapping) in order to obtain the most efficient optimization for group formation and task execution. Simulations results show that the use of overlapping population and fitness scaling present better results than non-overlapping population and unscaled fitness.
\end{abstract}

Keywords: Genetic algorithm, multi-robot systems, coordination.

\section{Introduction}

There are many fields where a single agent is not sufficient or enough to fulfill a determined task. Tasks like cleaning nuclear residuals, cleaning chemical accidents, forest fire combat or even on constructions, agriculture, hostile environment exploration, security and critical missions may be better accomplished when using a group of agents. Using robotic agents instead of human beings may add security, reliability and efficiency in these tasks. Multirobotic systems are extremely dependent on control techniques; they can add scalability, flexibility and robustness to a wide range of new applications [1]19], but they also bring a series of new questions to be solved in collaboration and cooperation. Specialized algorithms, composed by rules and automats have been developed seeking to coordinate these physical sets in dynamic environments, showing to be an extremely complex challenge [7/3. Due to this, a large number of researchers are 
migrating their efforts to several different approaches (e.g. application of classical intelligent artificial techniques, social models, market-based models, swarmbased models).

In the firefighting mission, one of the most important questions is related to the robot position setting. According to the actuation capability of each robot, weather condition (wind, rain), topography, and vegetation, several arrangements can be proposed. These arrangements, when suggested by a specialist, may not take in account a large number of variables. In these cases, machine learning techniques may be successfully used. One of the machine learning techniques that has been showing satisfactory results in solving optimization problems are Genetic Algorithms 1116. It consists in a global optimization algorithm that employs parallel and structured search strategies, directed by fitness points seeking, allowing the multi-criteria search in a multidimensional space. As it is an unsupervised technique, GAs don't need any previous information or solution database. Monitoring and combating of forest fire is an example of multirobotic system that could considerably reduces human, material and environmental losses.

In [21] we proposed and evaluated a Genetic Algorithm to accomplish the formation of a robotic squad that should perform a firefighting task; we evaluate characteristics like chromosome structure, mutation rate, types of mutation, number of individuals and crossover rate. However, two factors that has a strongly influence in the efficiency of the genetic search were not examined, which are: overlapping populations and scaling fitness. Thus, in this paper, we extended the work 21] to evaluate the use of overlapping populations and scaling fitness. The goal of this paper is to find a new set of parameters that allows that the GA converges more efficiently.

This paper has the following structure: Section 2 introduces short theoretical description of robot's applications. Section 3 presents concepts and applications of GAs. In Section 4 we explain the developed environment, the proposed fitness and the chromosome's structure. Section 5 describes the evaluation of all performed experiments. We finalize presenting the conclusion of the presented work and the future perspectives.

\section{Mobile Robotics}

Several current works demonstrate mobile robotic usage as individual systems on hostile operations as the rescue auxiliary robot Raposa [13] and SACI robot 14 developed for acting on fire combat. Moreover, there are robots to perform tasks on aquatic environments, space, caves and volcanoes exploration, and even to household use. Multirobotic systems must be formed by robots that are able to effective act on tasks, so knowledge about robotic control is a very important field. Works describing intelligent robot navigation can be seen in 2510. In 2004 and 2005, DARPA Grand Challenge [4], financed by the Defense Advanced Research Projects Agency organized a competition where the goal was building a completely autonomous vehicle that could complete a long way on dirt road on limited time. In 2007 the focus of the competition has changed. Renamed to 
DARPA Urban Challenge, it had a new goal to develop a vehicle that could perform autonomously on urban traffic, and execute tasks like parking, overtaking and intersection negotiations. These examples show trends in cooperation and multiple interactions.

The work with groups adds a great number of possibilities on tasking-solving but brings a series of new questions to be solved in collaboration and cooperation. Works using multirobotic systems like 24,2] uses pre-programmed rules on agents to perform formation. In [176] techniques are explored to perform works with collectives robotics, used mainly for the purpose of applying the concept of self-organization and collective optimization although task division is not directed explored. The works described in this section demonstrate that the application of mobile robotics in control of incidents is an important and active topic of research and development. These several competitions also demonstrate that there is still not a definitive or more adequate solution to the problem, and it is an open research field. In all consulted documents there is no consensual form to multirobotic system's conformation and actuation. Unpredicted situations with large degree of autonomy and robustness are still difficult to handle.

\section{Genetic Algorithms}

Genetic Algorithms (GA) [116 are global optimization techniques that employ parallel and structured search strategies, allowing multi-criteria search in a multi-dimensional space. They are methods classified as unsupervised, being unnecessary any previous information database. The GAs use iterative procedures that simulate the evolution process of a population constituted by candidate solutions of a certain problem. The evolution process is guided by a selection mechanism based on fitness of individual structures. For each algorithm iteration (single generation), a new structure set is created by information changing (bit or blocks) between selected structures of the previous generation [8]. The result of this conduces to the increasing individual fitness. A GA is structured in a way that the information about a determined system can be coded similarly to a biological chromosome, like a value array, where usually each sequence fragment represents a variable.

Dealing with applications, [18] uses a GA to satisfactorily optimize trajectory planning for a robot arm. In [9], a GA model correctly evolves values for force and time application to allow a robot to walk. The work [26] presents a GA model to evolve the exploration method of a mobile robot in an unknown environment. None of the works describes the use of overlapping population or fitness scaling. The works $[12 \mid 23$ show the use of overlapping population and fitness scaling in some theoretical problems (mathematical functions). Their results emphasize that the use of this issues are problem-dependent.

\subsection{Genetic Issues}

There are two important issues in the GA searching and optimization process: population diversity and selective pressure. These two issues are strongly related 
and have direct impact on the search efficiency. An increase in the selective pressure decrease the population diversity and vice-versa [15]. The population diversity and selective pressure has direct impact on premature convergence or ineffective search. Thus, it is important to stipulate a balance between these factors. Sampling mechanisms are attempt to achieve this goal [15]23]. Two factors that have directly influence in the sampling mechanisms are overlapping populations and fitness scaling.

The technique of overlapping populations consists of creating a copy of the current population. The genetic operations (selection, crossover and mutation) are carried out on this copy. After the evaluation of individuals in this cloned population, the individuals of original population are replaced considering a percentage of overlap defined by the user. The worst individuals of the original population are replaced by the best cloned population. Thus, they are kept in the original population of a certain amount of unique individuals. The $n$ best individuals have at least one more generation to accomplish for crossover and mutation. Replacing just one part of the population decreases the diversity of the population, thus the use of overlapping population converges faster. However, the exploitation of the search space is much more restricted [5]8].

Fitness scaling is done by applying a mathematical function on the original fitness, such that a sample of the population considers the scaled value instead of the raw fitness 23 . There are fitness functions where the value can not be really significant for the search with the GA. Thus, methods have been proposed in literature of scale for the fitness in the search that may increase or not selective pressure and consequently decrease or increase in the diversity of the population [8 15 23. In this study, beyond the evaluation of the raw fitness, four types of scaling are evaluated:

- Linear Scaling: Normalizes the fitness based on minimum and maximum fitness in current population. Sometimes can have a scale factor. The scale factor of 1.2 has been used in accordance to [8;

- Power Law Scaling: Maps objective scores to fitness scores using an exponential relationship defined as $f_{i}^{\prime}=f_{i}^{k}$, such that $f_{i}^{\prime}$ is the original fitness and $f_{i}^{\prime}$ is the scaled fitness. Several studies have shown success using $\mathrm{k}=$ 1.005, so we use these values in our search;

- Sigma Truncation Scaling: Normalizes using population mean and standard deviation, as $f_{i}^{\prime}=f_{i}+(\bar{f}-c \cdot \sigma)$. We use $c=2.0$ in accordance with specified in [22];

- Sharing Scaling: Reduces fitness for individuals that are similar to other individuals in the population. This scaling method is used to do speciation 1 . The fitness score is derived from its objective score by comparing the individual against the other individuals in the population. If there are other similar individuals then the fitness is derated [22].

\footnotetext{
${ }^{1}$ Speciation is the evolutionary process by which new biological species arise.
} 


\section{Group Formation}

In order to build a real physical implementation of robotic system, it is highly recommended to test the algorithms on virtual realistic simulation environments. Robotic system's simulation is specially necessary in case of large, expensive or fragile robots because it is an powerful tool to avoid wasting resources [7. In our case, the proposed simulator should be able to reproduce an environmental disaster for a multirobotic system actuation. We propose the situation of a forest fire. In this case a intelligent vehicle squad (as road grader) has the purpose of combating the forest fire acting by creating firebreaks around the fire.

The detailed characteristics of the fire spreading modeled to this work, as well as the forest fuel models and the real operation techniques are compiled into [20]. The simulated terrain is based on topographical maps and on forest fuel maps models that can also be seen in [20. The fire spreading simulation try to model the fire propagation as realistic as possible (considering present vegetation type, terrain slope, wind orientation and intensity).

Table 1. Chromosome structure (group of four robots)

\begin{tabular}{clcc}
\hline Gene & \multicolumn{1}{c}{ Function } & Min. value & Max. value \\
\hline 0 & Initial angle of robot 0 & $0.0^{\circ}$ & $360.0^{\circ}$ \\
1 & Final angle of robot 0; initial of robot 1 & $0.0^{\circ}$ & $360.0^{\circ}$ \\
2 & Final angle of robot 1; initial of robot 2 & $0.0^{\circ}$ & $360.0^{\circ}$ \\
3 & Final angle of robot 2; initial of robot 3 & $0.0^{\circ}$ & $360.0^{\circ}$ \\
4 & Final angle of robot 3 & $0.0^{\circ}$ & $360.0^{\circ}$ \\
5 & Initial radius of robot 0 & $10.0 \mathrm{~m}$ & $100.0 \mathrm{~m}$ \\
6 & Final radius of robot 0; initial of robot 1 & $10.0 \mathrm{~m}$ & $100.0 \mathrm{~m}$ \\
7 & Final radius of robot 1; initial of robot 2 & $10.0 \mathrm{~m}$ & $100.0 \mathrm{~m}$ \\
8 & Final radius of robot 2; initial of robot 3 & $10.0 \mathrm{~m}$ & $100.0 \mathrm{~m}$ \\
9 & Final radius of robot 3 & $10.0 \mathrm{~m}$ & $100.0 \mathrm{~m}$ \\
\hline
\end{tabular}

The planning mechanism uses a GA to define the initial and final operation positions of each robot for fire-fighting, which is developed using GAlib library 22. Considering that the combat agents are graders which have the finality to create firebreaks, we require that the GA returns the following information: initial and final angle, and initial and final radius for each robot, both related to the fire starting point. The proposed chromosome can be seen in the Table 1. In this, it is presented information of all group of involved agents, thus, the chromosome size depends on the number of robots in the system. Regarding the GAs parameters, we use alleles that limit the value set generated for each attribute (radius between 10.0 and 100.0 and angles between 0.0 and 360.0 degrees). The use of alleles reduces the search space. Also, we used real genome, optimized for floating point numbers.

The coordinates of operation are calculated using $x_{d}=x_{a}+r_{i} \cdot \cos \left(a_{i}\right)$ and $y_{d}=y_{a}+r_{i} \cdot \sin \left(a_{i}\right)$. Where $\left(x_{d}, y_{d}\right)$ is the robot's destination position, $\left(x_{a}, y_{a}\right)$ is the starting position of the fire, $r_{i}$ is the radius (gene 5 to 9 ) and $a_{i}$ is the angle (gene 0 to 4 ). The radius and the angle are defined specifically to each operation of each robot (initial and final coordinate of firebreaks creation). 
The proposed fitness is related with saved vegetation area and combat units usage rate; therefore, the fitness accumulates: (i) Total burned area: trying to minimize burned area, (ii) Firebreak total area: trying to minimize robot's work area, avoiding to create firebreak on non-risk areas, (iii) Trying to minimize the difference among general average of useful firebreaks in relation to each individual useful firebreak, equalizing worked areas. The GA tries to minimize the fitness function value, that means less burned vegetation, less created firebreaks, and less difference between the size of firebreaks of each robot.

\section{Experiments and Results}

We evaluated the system considering different percentages of overlap as well as different scaling methods, as show Table 2. Another GA parameters were obtained from [21]: \{selection by stochastic remainder sampling selector; uniform mutation; $10 \%$ of mutation; $90 \%$ of crossover; two point crossover; 150 individuals; 700 generations $\}$. The climatic characteristics of the fire simulation and the initial positions of robots are set as fixed in order to make the simulations. Ten simulations were performed with each set of parameters, totaling 200 simulations. The simulations considered the existence of four combat agents.

Table 2. Parameter set evaluated on the GA

\begin{tabular}{ccc}
\hline Set & Scaling Method & Overlapping \\
\hline$A_{1}-A_{4}$ & No Scaling & $\{$ No $; 25 \% ; 50 \% ; 75 \%\}$ \\
$B_{1}-B_{4}$ & Linear & $\{$ No $; 25 \% ; 50 \% ; 75 \%\}$ \\
$C_{1}-C_{4}$ & Sigma & $\{$ No $; 25 \% ; 50 \% ; 75 \%\}$ \\
$D_{1}-D_{4}$ & Power & $\{$ No $; 25 \% ; 50 \% ; 75 \%\}$ \\
$E_{1}-E_{4}$ & Sharing & $\{$ No $; 25 \% ; 50 \% ; 75 \%\}$ \\
\hline
\end{tabular}

Simulations results can bee seen in Fig. 1, Many visual observations were done on the fire simulator (using the results of the GA), showing that fitness that are below 3,500 are considered satisfactory - the simulations with fitness below 3,500 are able to extinguish the fire with well distributed areas among the robots. Fig.2 shows some satisfactory and unsatisfactory results of the simulations, where the Fig. 2(a) and 2(b) presents, respectively, fitness of 4,800 and 6,100; considered unsatisfactory results. The Fig. 2(c) present fitness of 3,200 units; considered satisfactory. We can see in Fig. 1 that only four sets present mean and standard deviation, for all simulations, below 3,500 units. These sets were: \{linear scaling with $50 \%$ of overlapping; sharing scaling with $50 \%$ of overlapping; no scaling with $75 \%$ of overlapping and sharing scaling with $75 \%$ of overlapping . Also, we can see that simulations that do not use overlapping populations present considerably worse values.

Whereas we obtained four sets with satisfactory results (fitness below 3,500), the next step was to seek improvements by reducing the number of individuals. Thus we performed a new set of simulations considering the four best sets of parameters but with a decrease in the number of individuals from 150 to 100 and 50. The size of the population is one of the most important choices faced by 


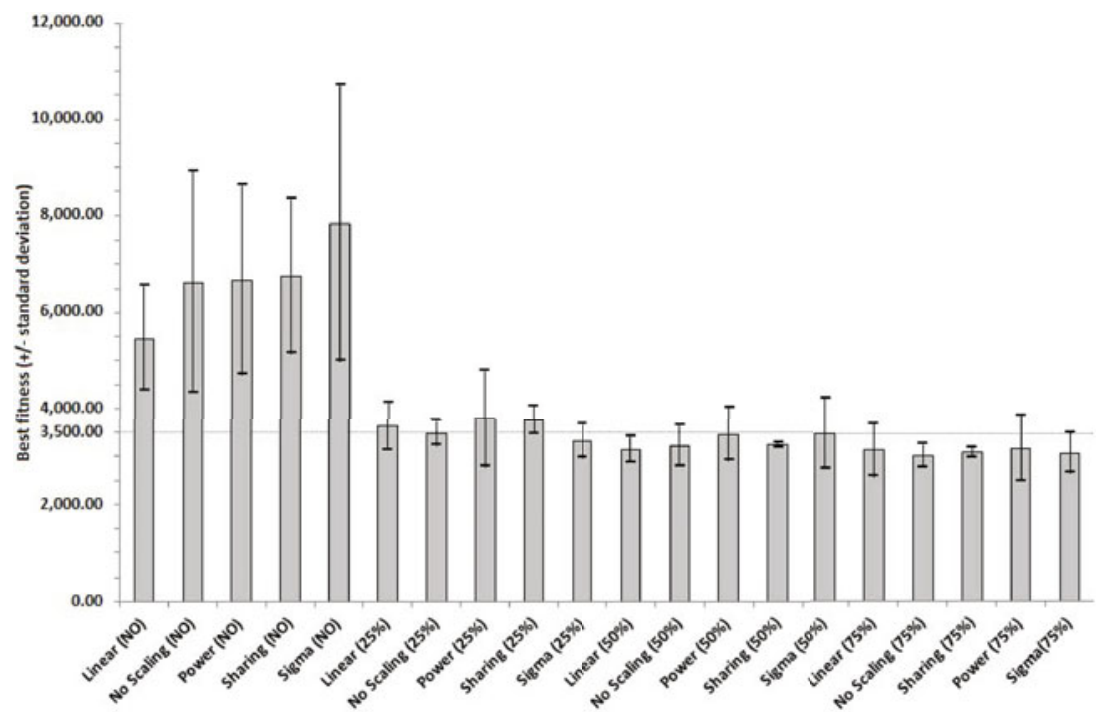

Fig. 1. Results of the evaluations presented in Table 2. The $\mathrm{x}$-axis shows the description of the experiment as (scaling method; percentage of overlapping).

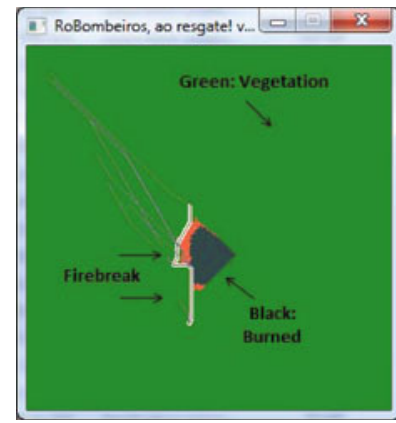

(a)

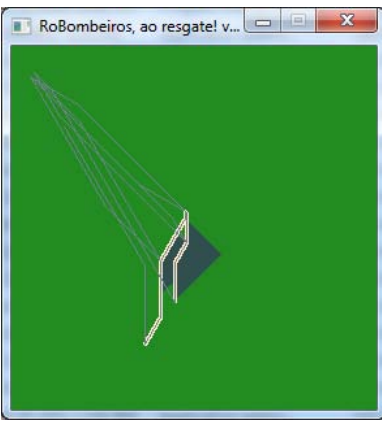

(b)

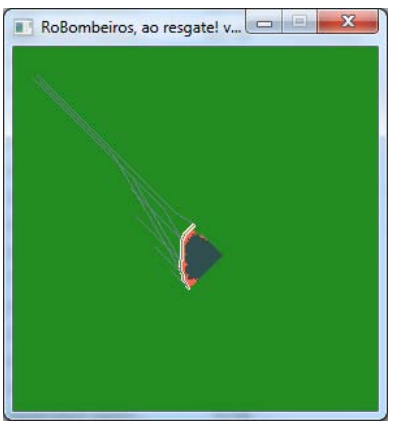

(c)

Fig. 2. (a) and (b) Unsatisfactory results. (a) Non overlapping with sigma scaling. (b) Non overlapping with no scaling. The firebreak are too large relative to what wold be necessary and are not well distributed among the robots. (c) Satisfactory result using sharing scaling and $75 \%$ of overlapping.

any user of GA [15. If the population size is too small, the GA may converge too fast (with unsatisfactory result). If the population is too large, there may be a waste of computational resources.

We can see in Fig. 3(a) that decreasing in the number of individuals, the simulations results are worse. However, even using 100 individual instead of 150, one parameter set could obtain all results with fitness below 3,500 units. This parameter set is that used $75 \%$ of overlapping and sharing scaling. No other set 


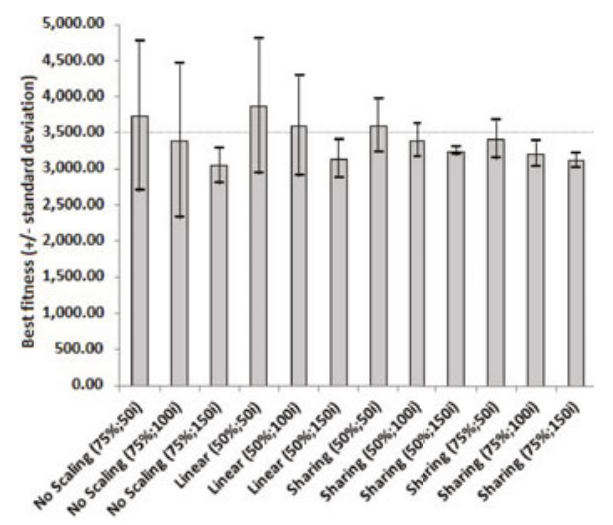

(a)

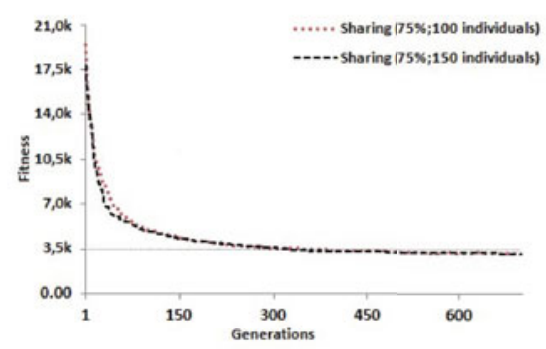

(b)

Fig. 3. (a) Results of the evaluations considering the four best sets from Fig. 1 but using different populations size. The x-axis shows the parameter set as (scaling method; percentage of overlapping; number of individuals (b) Evolution of fitness according to number of generations, with variable population size.

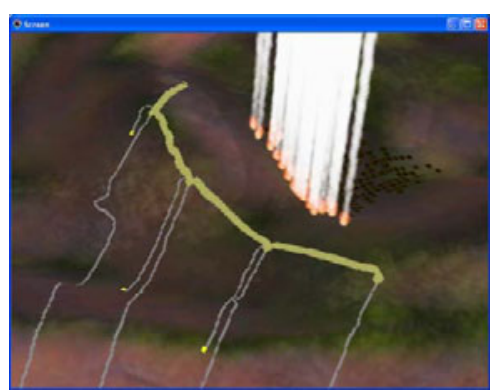

(a)

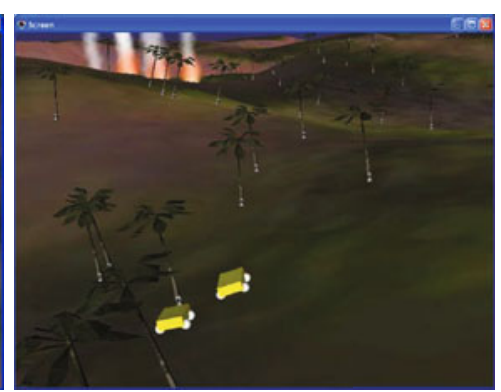

(b)

Fig. 4. (a) Four robots creating a firebreak. (b) Detailed view of mobile robots moving to the fire.

could obtain all simulations below 3,500 using 100 individuals. Fig. 3(b) present the fitness curve considering the best set described on previous paragraph. We can see that using both 100 or 150 individuals, the curves are quite similar.

Fire spread simulation considered East-West wind direction and relative wind speed at $7 \mathrm{~km} / \mathrm{h}$; robot navigation speed of $35 \mathrm{~km} / \mathrm{h}$; robots positioned on $2 \mathrm{~km}$ far from fire threshold base. The simulations results show that, for the proposed fitness function and chromosome structure, the use of $75 \%$ of percentage of overlapping and sharing scaling allowed to obtain best results. Fig. 4 presents satisfactory evolution result applied on $3 \mathrm{D}$ virtual simulation environment. The $3 \mathrm{D}$ prototype showed that robots completely surround the fire and create the firebreaks on a satisfactory way. 


\section{Conclusions and Future Work}

In this paper we evaluate the use of overlapping populations and fitness scaling in a GA applied to multi-robot squad formation and coordination. The robotic task is performed over a natural disaster scenario (a forest fire simulation). The robot squad mission is surrounding the fire and avoiding fire's propagation based on the strategy proposed by the GA. Simulations have been carried out to evaluate the effect of fitness scaling and overlapping population in the efficiency of GA search. The simulation's results shows that the use of overlapping population and fitness scaling presents better results than non-overlapping population and unscaled fitness.

There are three future work planned: (i) adaptations to the model to allow experiments using real robots; (ii) the theoretical mathematical study about the influences of fitness scaling using the proposed chromosome; (iii) evaluations of scalability and flexibility of the proposed group formation model.

\section{Acknowledgements}

The authors acknowledge the support granted by CNPq and FAPESP to the INCT-SEC (National Institute of Science and Technology - Critical Embedded Systems - Brazil), processes 573963/2008-8 and 08/57870-9. Also acknowledge CAPES and FAPESP for their financial support of this research (doctoral grant).

\section{References}

1. Arai, T., Pagello, E., Parker, L.: Guest editorial: advances in multirobot systems. IEEE Transactions on Robotics and Automation 18(5), 655-661 (2002)

2. Balch, T., Arkin, R.C.: Behavior-based formation control for multi-robot teams. IEEE Transactions on Robotics and Automation 14(6), 926-939 (1998)

3. Bekey, G.A.: Autonomous Robots: From Biological Inspiration to Implementation and Control. The MIT Press, Cambridge (2005)

4. Darpa: Darpa grand challenge webpage (2007), http://www.darpa.mil/grandchallenge

5. DeJong, K.A.: An analysis of the behavior of a class of genetic adaptive systems. Ph.D. thesis (1975)

6. Dorigo, M., Trianni, V., Sahin, E., Gro, R., Labella, T., Baldassarre, G., Nolfi, S., Deneubourg, J., Mondada, F., Floreano, D., Gambardella, L.: Evolving selforganizing behaviors for a swarm-bot. Autonomous Robots 17, 223-245 (2004)

7. Go, J., Browning, B., Veloso, M.: Accurate and flexible simulation for dynamic, vision-centric robots. In: Proceedings of 3th International Joint Conference on Autonomous Agents and Multi-Agent Systems, AAMAS 2004 (2004)

8. Goldberg, D.E.: Genetic Algorithms in Search, Optimization and Machine Learning. Addison-Wesley Professional, Reading (1989)

9. Heinen, M.R., Osório, F.S.: Applying genetic algorithms to control gait of simulated robots. In: Electronics, Robotics and Automotive Mechanics Conference (2007)

10. Heinen, M., Osório, F., Heinen, F., Kelber, C.: Seva3d: Using artificial neural networks to autonomous vehicle parking control. In: International Joint Conference on Neural Networks, IJCNN 2006 (2006) 
11. Holland, J.H.: Adaptation in Natural and Artificial Systems. University of Michigan Press, Ann Arbor (1975)

12. Hopgood, A.A., Mierzejewska, A.: Transform ranking: a new method of fitness scaling in genetic algorithms. In: Proc. AI 2008, Research and Development in Intelligent Systems XXV (2008)

13. IdMind: Projecto raposa (2009), http://raposa.idmind.pt

14. Macedo, A.R.M., Macedo, A.R.L., Duarte, J.B.F.: Robótica aplicada ao combate a incidentes. Revista TN Petróleo (53), 108-113 (2007)

15. Michalewicz, Z.: Genetic Algorithms + Data Structures = Evolution Programs. Springer, Heidelberg (1996)

16. Mitchell, M.: An Introduction to Genetic Algorithms. The MIT Press, Cambridge (1996)

17. Mondada, F., Gambardella, L.M., Floreano, D., Nolfi, S., Deneubourg, J., Dorigo, M.: The cooperation of swarm-bots: physical interactions in collective robotics. IEEE Robotics \& Automation Magazine 12(2), 21-28 (2005)

18. Nguyen, V.B., Morris, A.S.: Genetic algorithm tuned fuzzy logic controller for a robot arm with two-link flexibility and two-joint elasticity. Journal of Intelligent and Robotic Systems 49(1), 3-18 (2007)

19. Parker, L.E.: Distributed intelligence: Overview of the field and its application in multi-robot systems. Journal of Physical Agents 2(1), 5-14 (2008)

20. Pessin, G.: Evolução de Estratégias e Controle Inteligente em Sistemas MultiRobóticos Robustos. Master's thesis, Universidade do Vale do Rio dos Sinos (2008)

21. Pessin, G., Osório, F.: Avaliação de um modelo tolerante a falhas para atuação tática de grupos robóticos utilizando algoritmos genéticos. In: XXXV Conferencia Latinoamericana de Informática (CLEI 2009), pp. 1-10 (2009)

22. Wall, M.: Galib - a c++ library of genetic algorithm components. Massachusetts Institute of Technology (1996)

23. Whitley, D.: The genitor algorithm and selection pressure: why rank-based allocation of reproductive trials is best source. In: Proceedings of the Third International Conference on Genetic Algorithms, pp. 116-121 (1989)

24. Yamaguchi, H.: Adaptive formation control for distributed autonomous mobile robotgroups. In: IEEE Int. Conf. on Robotics and Automation (1997)

25. Zhao, Y., Collins, E.G.: Robust automatic parallel parking in tight spaces via fuzzy logic. Robotics and Autonomous Systems 51, 111-127 (2005)

26. Zhou, Y.: An Area Exploration Strategy Evolved by Genetic Algorithm. Master's thesis, University of Georgia (2005) 